\title{
Biopsy navigator: a smart haptic interface for interventional radiological gestures
}

\author{
Gaëtan Marti, Patrice Rouiller, Sébastien Grange, Charles Baur \\ Swiss Federal Institute of Technology (EPFL) \\ IPR / LSRO / VRAI Group \\ 1015 Lausanne, Switzerland
}

\begin{abstract}
The VRAI group at EPFL is conducting research in the fields of virtual reality and haptics (force-feedback) for medical applications. In particular, we have developed visualization techniques for medical images from various sources, and a high-performance haptic interface. In this paper, we present a technique that combines visualization with haptic rendering in order to provide real-time assistance to medical gestures. To demonstrate this technique, we have developed the BiopsyNavigator, a system that provides haptic feedback to the surgeon using patient specific data. Before the biopsy, it provides the surgeon with the ability to simulate the intervention. During the biopsy, haptic feedback is used to first help the surgeon to find the target and to define the optimal trajectory, then to physically guide the surgical gesture along the chosen path. Finally, haptic information is used to indicate that the target has been reached. Future developments will include real-time update of the patient model from various sources, including $\mathrm{C}$-arm mounted $\mathrm{CT}$ and ultrasonic probes.
\end{abstract}

Keywords: haptic-based biopsy navigation, haptic guidance

\section{Introduction}

Biopsy practice relies heavily on the manual dexterity and experience of medical personnel. In the field of ultrasound-guided biopsy for example, significant experience is required to perform error-free and painless intervention [1]. This experience can be partly acquired using simulators. A recent study [6], demonstrated that using haptic (force-feedback) and visual cues in needle insertion tasks improves 3D navigation performance. This trend is further confirmed by numerous recent research developments [2,3,4,5]. Robotics and image-guided biopsy have become important aids to assist medical personnel during the act. In the TRUS system [7], a robot is used to accurately position a needle in 3D-space and to obtain a sample of the prostate. The main advantages of this robot are accuracy and reliability but, unfortunately, broad clinical use of this system is unlikely due to the expense and setup time [8]. Image-guided biopsy navigation systems $[9,10]$ match operative data (MRI, US) with needle's position. These systems improve safety and accuracy during the insertion but do not consider insertion speed, which is important because a slow insertion is painful for the patient and more traumatic than a rapid insertion. Yet, if the physician inserts the needle too fast it is possible that it will push it too far into the patient's tissue. 
To address these concerns, we propose a needle insertion simulator and a CT / ultrasound (US) navigation system combined with a haptic device. In our system, the needle is directly connected to the haptic apparatus. During the procedure, the physician can freely use the device to localize the biopsy entry point, as well as, to provide guidance during needle insertion. We believe that by using a force-feedback device during the medical act, speed, safety and accuracy especially in the 3D space can all be improved.

\section{Method}

The BiopsyNavigator is a combination of haptic hardware and virtual reality software that enables the surgeon to benefit from CT/US data during biopsy procedure. The data is used for both targeting and guidance.

The system's hardware consists of a biopsy needle that is mounted on a 6 degree-of-freedom Delta Haptic Device (3 degrees-of-freedom in translation, and 3 degrees-of-freedom in rotation around the user's wrist) [11], as illustrated in Figure 1. A needle holder has been adapted on this commercially available force-feedback device (Figure 1b).

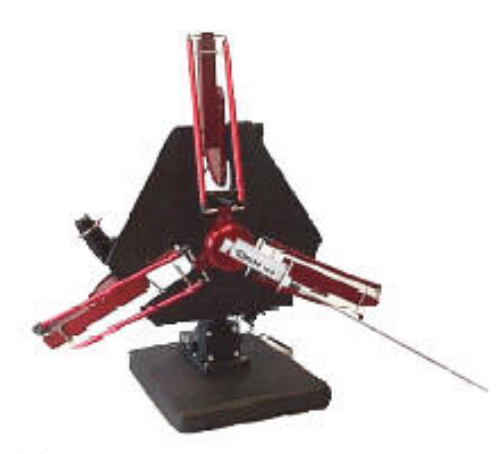

(a)

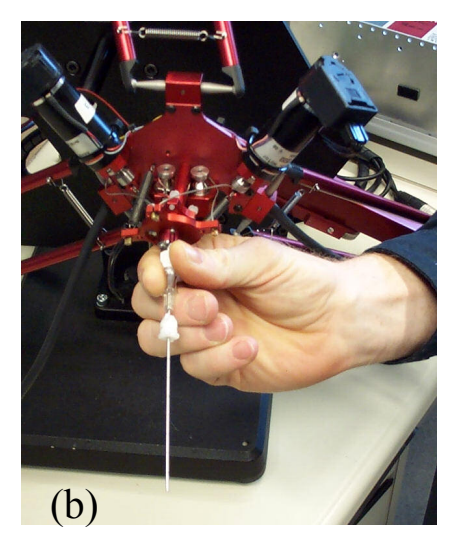

(b)

Fig. 1: Hardware setup: (a) Delta Haptic Device (6-DOF) with a biopsy needle, (b) Detail view on the needle holder.

The software consists of a preoperative interface and a navigation interface. The first interface allows patient registration and data loading. The second interface provides a 3D overview display that illustrates the position of the needle relative to the dataset (Figure 2), a slice view and additional information such as the distance of the needle tip to the target area. The CT/US scan data is interpolated over the scanned volume, so that the $3 \mathrm{D}$ virtual representation on the GUI allows the user to visualize any cut plane through the CT/US scan volume in the slice view (Figures $2 b$ and $3 b$ ). During the procedure, the visualization plane on the GUI is defined by the biopsy needle position and orientation. 


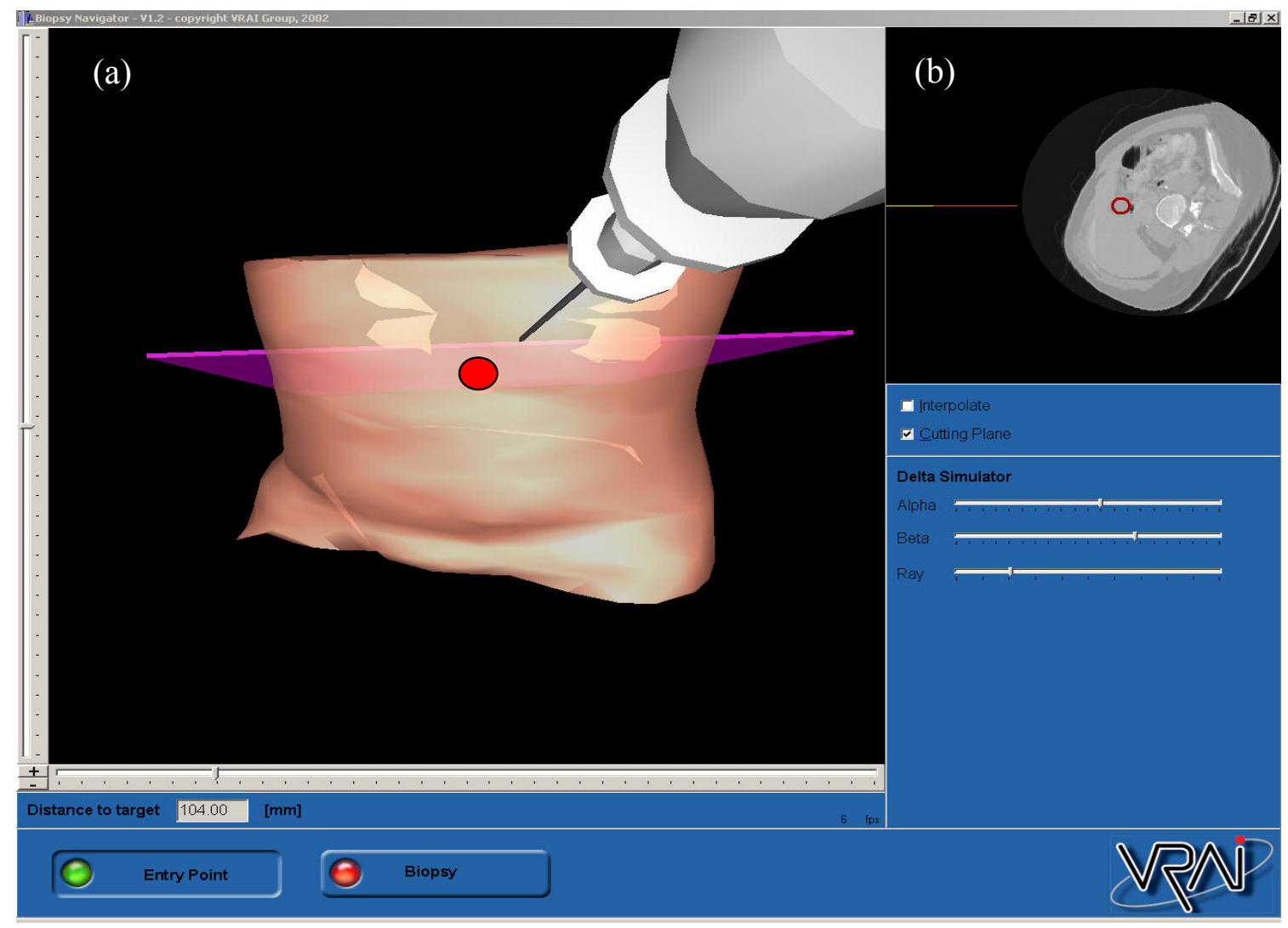

Fig. 2: Navigation interface available during the procedure. (a) 3D display representing the needle and the position of the slice relatively to the patient. The target biopsy area is the circle, (b) Slice view. The ring is biopsy area. The line represents the position of the needle.

The procedure is performed using the BiopsyNavigator as shown in Figure 3a. There are three phases in the intervention and optional simulation step.

\section{Phase 1: data loading and registration}

After a 3D scanner exam (CT, US) of the patient has been performed, volume data are loaded in the navigation software. An interface allows the physician to segment the biopsy area in the slices. 3D models of skin and the target area are automatically constructed for further visualization and guidance help.

In the current implementation of the system, the translation of the scanner's table is used for registration. This means that the patient must not move between the exam and the biopsy. An extension with continuous tracking and registration using some dedicated tools such as the low-cost tracker developed at the VRAI group is ongoing.

\section{Phase 2: insertion path definition}

During this phase, the surgeon uses the BiopsyNavigator to define the most appropriate insertion path in the patient for the needle. Although the operator is free to move the needle attached on the haptic device, the hardware constrains the orientation of the needle so that it 
always points towards the target, as defined in the previous step. Applying a force that exceeds a predefined threshold can nevertheless, modify preferential alignment. The virtual representation on the GUI, interpolated in real-time from the latest set of CT scans, makes it possible to visually validate the direction of the needle. The user can decide by manipulating the needle and by visualizing the trajectory whether or not, the access path is affecting areas that should be avoided (e.g. areas that are hazardous for the patient or that present too much mechanical resistance). Figure $3 \mathrm{~b}$ shows the trajectory information (slice view) that is displayed in real-time to the surgeon.

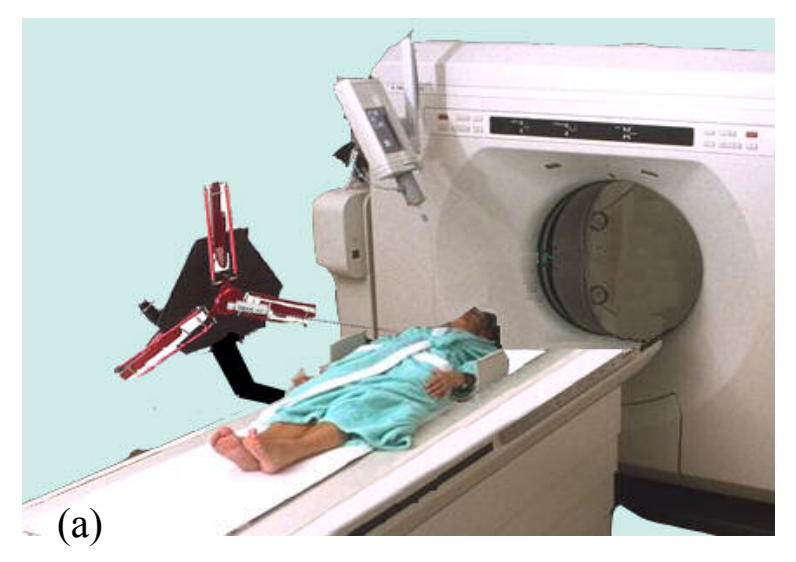

(a)

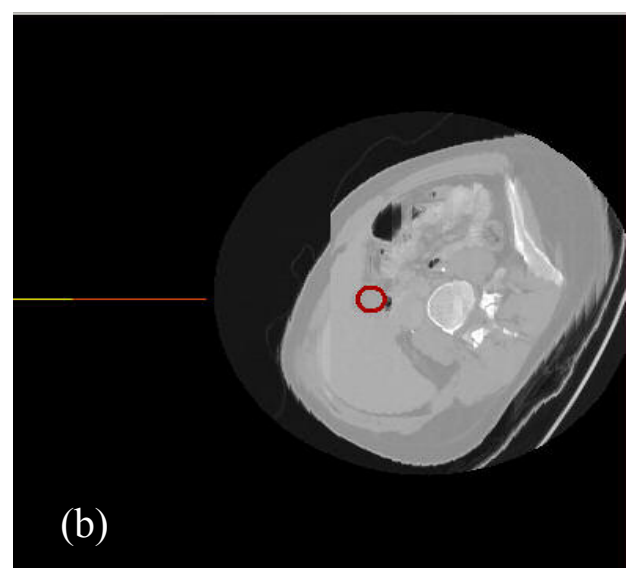

Fig. 3: (a) Operating setup. The haptic device is attached to the scanner's table. Registration is performed using translation. (b) Slice view allowing real time trajectory indication.

\section{Phase 3: path guidance}

Once the optimal entry trajectory is defined, the system is set to "biopsy" mode. In this mode, the haptic device only allows movements along the rectilinear trajectory defined during phase 2. This natural guides the needle along the defined axis towards the target. The user can, however, modify the constrained axis at any time by applying a force greater than a predefined threshold. Once the target is reached, the haptic device conveys a spring-like resistance to the surgeon's hand to indicate that the needle is in place. Again, the surgeon can correct the final position by applying a force above a user-adjustable threshold.

\section{Simulation phase}

The system can also be used to simulate patient-specific biopsy before the intervention. The system works in much the same way, expect that during phase 3 , a resistance specific to each tissue and the interface between tissues as measured from the CT scan is translated into tactile information. The goal is to validate the planned gesture as well as to train for a specific operation. Prior experience demonstrates that training involving both haptic and visual feedback provides better efficiency results. 


\section{Results}

Our existing system does not address "real" registration of CT images with the patient and the force feedback system. Specifically, two assumptions are made. First, we assume that the patient position does not change between the time the CT scan is performed, and the time of the biopsy procedure. The second assumption is that the haptic device is attached to the patient's table. These assumptions, in particular the first, are obviously not realistic from a clinical perspective.

From a review with several physicians, the following remarks were collected:

- Tactile guidance and feedback is of real interest for such procedures.

- After the scanning phase, tissue deformation due to breath or patient displacement should be handled. The use of interventional modalities such as C-Arm or US decreases this kind of error because the delay between acquisition and needle insertion is shorter.

- Installation of the haptic system in the workspace (i.e. the operation area) and the ease of clipping the probe and needle tools into place must be evaluated. The same applies to the associated sterility requirements.

- The system is of limited interest as a training tool, with the exception of applications requiring cutting through hard structures.

\section{Conclusion}

We have developed a system that combines a visualization tool and a haptic interface to provide guidance during the execution of medical gestures. In particular, this system is designed to support biopsy by allowing the user to easily define the optimal trajectory to the biopsy target, and to be guided to the target. Clinical validation of the system is planned for 2003.

\section{Acknowledgements}

This research was performed in collaboration with the University Hospital of Geneva (HUG), Switzerland and was supported by a grant from the Swiss NSF Computer Aided and Image Guided Medical Interventions (NCCR CO-ME) program. 


\section{References}

1 J. Bleck, W. Wienhöfter: VR Trainer: Haptic Feedback for Ultrasound-guided Biopsy. Falk Symposium 124. Medical Imaging in Gastroenterology and Hepatology, 2001.

2 U. Kühnapfel. Force feedback device and KISMET visualisation tool for biopsy. http://iregt1.iai.fzk.de/VOLREN/volren_start.html, last updated march 1997.

3 T. Dang, T. M. Annaswamy, M. A., Srinivasan: Development and Evaluation of an Epidural Injection Simulator with Force Feedback for Medical Training. Studies in Health Technology and Informatics, Vol. 29, pp. 564-579, 1996.

4 D. S. Kwon, K. U. Kyung, S. M. Kwon, J. B. Ra, H. W. Park, H. S. Kang, J. Zeng, K. R. Cleary: Realistic Force Reflection in a Spine Biopsy Simulator. Proceedings of the IEEE International Conference on Robotics and Automation, pp. 1358-1363, 2001.

5 J. B. Ra, et al: A Visually Guided Spine Biopsy Simulator with Force Feedback. SPIE International Conference on Medical Imaging, pp. 36-45, 2001.

6 O. Gerovichev, P. Marayong, A. M. Okamura: The Effect of Visual and Haptic Feedback on Manual and Teleoperated Needle Insertion. MICCAI, 2002.

7 B. R. Lee, J. A. Cadeddu, D. Stoianovici, L.R. Kavoussi : Telemedicine and Surgical Robotics : Urologic Applications. Review in urology, Spring 1999.

8 A. Rovetta, R. Sala: Robotics and telerobotics applied to a prostate biopsy on a human patient. Proceedings of the Second International Symposium on Medical Robotics and Computer Assisted Surgery, Baltimore, Md, p 104, 1995.

9 K. Salisbury, D. Brock, T. Massie, N. Swarup, C. Zilles: Haptic Rendering: Programming Touch Interaction with Virtual Objects, Proceedings of the 1995 Symposium on Interactive 3D graphics, 1995.

10 N. Hata et al: MRI-guided prostate biopsy using surgical navigation software: device validation and preliminary experience, Radiology, 220(1), 263-8, 2001.

11 S. Grange, F. Conti, P. Helmer, P. Rouiller and C. Baur: Overview of the Delta Haptic Device. Eurohaptics '01, Birmingham, England, July 2001. 